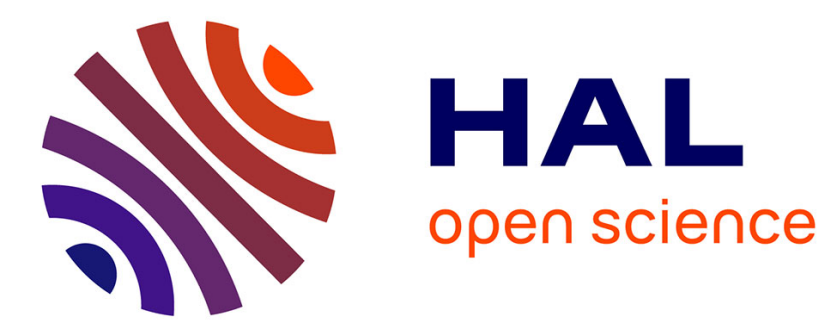

\title{
Réfrigération solaire par simulation en absorption sèche
}

J. Fléchon, F. Machizaud

\section{To cite this version:}

J. Fléchon, F. Machizaud. Réfrigération solaire par simulation en absorption sèche. Revue de Physique Appliquée, 1980, 15 (4), pp.909-918. 10.1051/rphysap:01980001504090900 . jpa-00244804

\section{HAL Id: jpa-00244804 https://hal.science/jpa-00244804}

Submitted on 1 Jan 1980

HAL is a multi-disciplinary open access archive for the deposit and dissemination of scientific research documents, whether they are published or not. The documents may come from teaching and research institutions in France or abroad, or from public or private research centers.
L'archive ouverte pluridisciplinaire HAL, est destinée au dépôt et à la diffusion de documents scientifiques de niveau recherche, publiés ou non, émanant des établissements d'enseignement et de recherche français ou étrangers, des laboratoires publics ou privés. 


\title{
Réfrigération solaire par simulation en absorption sèche
}

\author{
J. Fléchon et F. Machizaud \\ Laboratoire de Physique des Dépôts Métalliques, Université de Nancy-I \\ C.O. 140, 54037 Nancy Cedex, France
}

(Reçu le 16 mars 1979, révisé le 14 janvier 1980, accepté le 15 janvier 1980)

\begin{abstract}
Résumé. - Après avoir tracé dans une précédente publication [4] le réseau $(1 / T, \log P)$ du couple frigorigène $\mathrm{CaCl}_{2}-\mathrm{NH}_{3}$ pour diverses concentrations en ammoniac, nous soumettons le binaire, par simulation, à des conditions thermiques que lui imposerait le rayonnement solaire. Nous examinons ainsi la dissociation isotherme du matériau durant cinq heures correspondant à l'ensoleillement diurne, puis sa régénération à l'ambiante, associée à son comportement nocturne. Des considérations cinétiques et énergétiques permettent d'interpréter l'expérience.

Abstract. - After having defined in a previous publication [4], the network $(1 / T, \log P)$ of the frigorigene couple $\mathrm{CaCl}_{2}-\mathrm{NH}_{3}$ for different concentrations in ammonia, by simulation we submit the binary mixture to thermal conditions that would impose solar radiation upon it. We thus examine the isothermal dissociation of the material for 5 hours corresponding to daily insolation. Then its regeneration at ambiant temperature for the night. Kinetic and energy considerations allows us to interpret the experiment.
\end{abstract}

1. Objet de l'étude. - Nous recherchons un couple frigorigène adapté aux conditions climatiques difficiles des pays situés en zone tropicale. Ceci afin de définir dans quelle mesure l'énergie solaire permettrait, dans ces régions, la réalisation des réfrigérateurs exploitables pratiquement à l'aide d'absorption intermittente et associés à des capteurs plans dont nous avons fait une étude systématique [1, 2, 3].

Après avoir discuté des possibilités de différents couples [4], nous avons montré que, dans l'ambiance retenue, l'absorption sèche semblait la plus propre à l'obtention du froid solaire. Parmi les couples susceptibles d'un emploi efficace, $\mathrm{CaCl}_{2}, \mathrm{NH}_{3}$ a fait l'objet de réalisations domestiques en Allemagne $[5,6,7,8]$ et d'études en laboratoire pour l'exploitation de l'énergie solaire $[9,10,11]$.

Nous avons tracé [4] pour le binaire le réseau $(\log P, 1 / T)$ dans le domaine des concentrations comprises entre $\mathrm{CaCl}_{2}, 8 \mathrm{NH}_{3}$ et $\mathrm{CaCl}_{2}, 2 \mathrm{NH}_{3}$ soit en masse $55 \%$ et $26 \%$ puisqu'il n'est pas possible de dissocier l'ammoniacate à $2 \mathbf{N H}_{3}$ sans porter sa température au-delà de $150^{\circ} \mathrm{C}$ [12].

$\mathrm{Au}$ moment de passer à l'application pratique, c'est-à-dire d'envisager la réfrigération solaire, il conviendra de savoir si, par kilogramme de $\mathrm{CaCl}_{2}$, c'est effectivement de 1,51 de $\mathrm{NH}_{3}$ soit environ $900 \mathrm{~g}$ ou 270 kilofrigories dont on pourra disposer, ou bien, si la brièveté de l'insolation efficace et l'insuffi- sance du rayonnement global limiteront ces grandeurs et dans quelles proportions.

Après avoir défini les conditions de l'expérience, nous examinerons les résultats des études thermocinétique et énergétique.

2. Méthode expérimentale. - L'appareil utilisé (Fig. 9) en simulation au laboratoire est formé d'un générateur en acier essayé à 30 bars contenant deux kilogrammes de $\mathrm{CaCl}_{2}$, anhydre séché à l'étuve à $120^{\circ}$ durant plusieurs jours, réuni à un évaporateur par l'intermédiaire d'un condenseur refroidi à l'air. Les $6 \mathrm{NH}_{3}$ absorbables et libérables correspondent à 31 de liquide (densité 0,61), 11 restant combiné à $\mathrm{CaCl}_{2}$ dans les conditions de l'expérience. Le volume de frigorigène sera déterminé par simple lecture de niveau libre dans un tube de verre à l'épreuve des pressions de travail.

Compte tenu du but poursuivi et des conditions climatiques envisagées, il convient d'observer que l'insolation efficace ne dure jamais plus de 6 heures et par conséquent de simuler, à des températures du générateur variant de 70 à $140{ }^{\circ} \mathrm{C}$, l'action du rayonnement solaire par apport d'une énergie électrique convenable en mesurant le volume de $\mathrm{NH}_{3}$ liquide obtenu par dissociation des ammoniacates. Dans une première approche, on simplifiera l'étude du comportement diurne du générateur en le main- 
tenant durant 5 heures à une température constante comprise entre 70 et $140^{\circ} \mathrm{C}$. En effet, nos études précédentes sur les capteurs plans [3] ont montré qu'en fonction de la puissance radiative incidente leurs températures limites d'équilibre thermique étaient comprises dans ce domaine.

L'énergie électrique dissipée dans les résistances correspond à

$$
V_{\text {eff }}=220 \mathrm{~V}, \quad I_{\text {eff }}=4,3 \text { soit } P_{\text {moy }}=0,95 \mathrm{~kW},
$$

ce qui représente qualitativement l'énergie solaire incidente sur $1 \mathrm{~m}^{2} 25$ de capteur. Nous pourrons ensuite apprécier, sur une durée maximale de 18 heures, par enregistrement des températures et mesure des volumes de $\mathrm{NH}_{3}$ dans l'évaporateur, les effets thermiques associés à son évaporation nocturne pour reformer les ammoniacates, donc la capacité effective de réfrigération du couple.

Les variables sont : la température $T$ du générateur, la pression maximale de vapeur $P$ de $\mathrm{NH}_{3}$ partiellement condensé dans l'évaporateur, $C$ la concentration d'ammoniac dans l'ammoniacate partiellement dissocié. Cette dissociation est d'autant plus faible que $P_{\mathrm{m}}$ est élevé. $P_{\mathrm{m}}$ varie dans les conditions habituelles de 9 à 16 bars. En effet $P_{\mathrm{m}}$ est rigoureusement déterminé par la température de condensation de $\mathrm{NH}_{3}$. Or si l'on se réfère aux conditions climatiques de la zone tropicale, le condenseur dans lequel se liquéfie l'ammoniac est porté par la quantité de chaleur ainsi libérée à une température qui varie de l'ambiante à une dizaine de degrés au-dessus de celle-ci, soit environ $40{ }^{\circ} \mathrm{C}$. La courbe de vaporisation de $\mathrm{NH}_{3}$ (diagramme de Bosnjakovic et Wucherer [4]) montre que la pression $P_{\mathrm{m}} \simeq 16$ bars.

3. Etude thermocinétique. - 3.1 CoMportemENT DIURNE : DISSOCIATION ISOTHERME. - La figure 1 résume l'ensemble des expériences. Nous nous référons initialement à 3 cas essentiels pour la réfrigération :

Couple riche en $\mathrm{NH}_{3}$ : $\mathrm{CaCl}_{2}, 8 \mathrm{NH}_{3}$ octoammoniacate

Couple moyennement riche : $\mathrm{CaCl}_{2}, 6 \mathrm{NH}_{3}$ hexaammoniacate

Couple pauvre

: $\mathrm{CaCl}_{2}, 4 \mathrm{NH}_{3}$ tétraammoniacate.

3.1.1 Observations. - La courbe (a) précise, dans les conditions de travail, la limite de dissociation des ammoniacates après un temps infini. Elle se déduit en effet du réseau $(1 / T, \log P)$ précédemment tracé [4] et qui indique sans ambiguité, la relation entre $P_{\mathrm{m}}, T$ et $C$ à l'équilibre, c'est-à-dire après un temps infini. Les volumes portés en ordonnée sur la figure 1 explicitent $C$ puisque nous disposons dans l'expérience de $2 \mathrm{~kg}$ de $\mathrm{CaCl}_{2}$ et de $31 \mathrm{~d}$ 'ammoniac liquide. Cette courbe (a) montre qu'en dessous de $100^{\circ}$, il ne saurait être question d'atteindre le diammoniacate en fin d'opération, quelle que soit

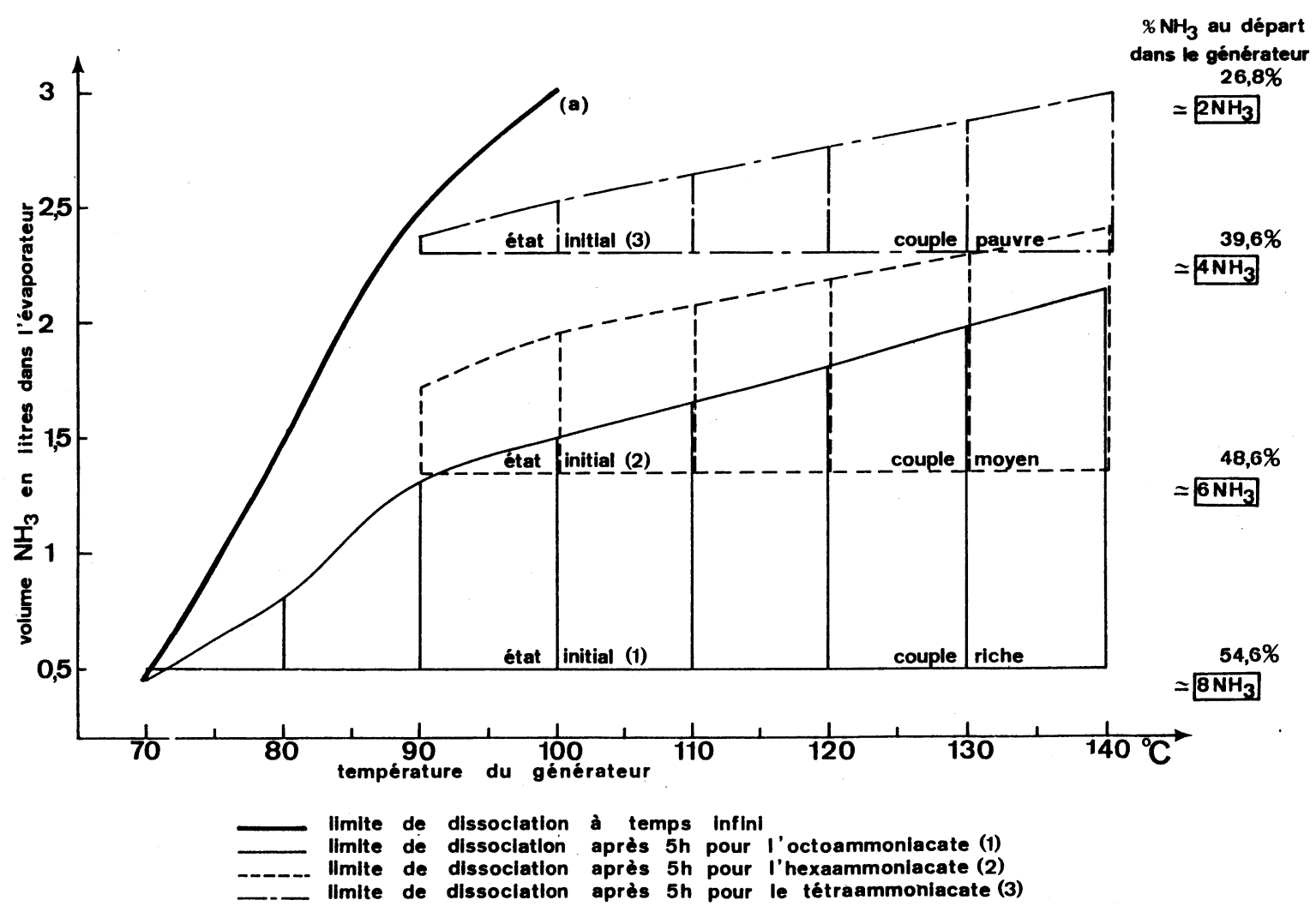

Fig. 1. - Dissociation diurne isotherme des ammoniacates en 5 heures de chauffe.

[Diurnal and isotherm dissociation of the ammoniacates in 5 hours of furnace.] 
la durée de chauffe, et, jusqu'à $70^{\circ} \mathrm{C}$, la dissociation des ammoniacates de $\mathrm{CaCl}_{2}$ est inexistante.

La conclusion pratique est qu'une réfrigération ne peut être envisagée par cette méthode si l'insolateur plan ne peut dépasser largement cette température.

De 70 à $90^{\circ} \mathrm{C}$, les résultats expérimentaux portés sur la figure 1 montrent que seul le couple initial riche peut libérer en 5 heures de 0 à 0,81 d'ammoniac liquide pour $2 \mathrm{~kg}$ de $\mathrm{CaCl}_{2}$ anhydre.

De 90 à $140^{\circ} \mathrm{C}$, la quantité de $\mathrm{NH}_{3}$ condensé, en plus de celle qui l'était à $90^{\circ} \mathrm{C}$, donc l'augmentation de la vitesse moyenne de dissociation des ammoniacates, est sensiblement proportionnelle à l'écart de température $(\theta-90)$ soit $0,751 / 50^{\circ}$, c'est-à-dire, en 5 heures, un gain de $0,151 / 10^{\circ}$ et ceci quelle que soit la richesse initiale du couple.

On peut le traduire, pour cette durée optimale de chauffe, soit par un gradient de dissociation $D$ en fonction de la température $\nabla_{T} D=5 \times 10^{-3} \mathrm{par}$ référence à la masse totale de $\mathrm{NH}_{3}$ libérable, soit par un taux effectif de $\mathrm{NH}_{3}$ libéré à une température donnée après 5 heures de chauffe isotherme.

3.1.2 Taux de $\mathrm{NH}_{3}$ libéré. - Le tableau I permet d'indiquer en fonction de $\theta$ pour les trois types de couples initiaux, le taux \% de $\mathrm{NH}_{3}$ libéré sous l'action de la chaleur après 5 heures de maintien isotherme du générateur.

Tableau I.

\section{$\theta$}

\begin{tabular}{crrrrrr} 
Couple initial & \multicolumn{1}{c}{90} & 100 & 110 & 120 & 130 & 140 \\
- & - & - & - & - & - & - \\
$\mathrm{CaCl}_{2}, 8 \mathrm{NH}_{3}(3)$ & 34 & 40 & 47 & 52 & 58 & 66 \\
$\mathrm{CaCl}_{2}, 6 \mathrm{NH}_{3}(2)$ & 15 & 24 & 29 & 33 & 37 & 42 \\
$\mathrm{CaCl}_{2}, 4 \mathrm{NH}_{3}(1)$ & 3 & 9 & 14 & 18 & 22 & 28
\end{tabular}

Notons que les masses de $\mathrm{NH}_{3}$ libérables correspondant à (3) sont le triple de celles liées à (1) et les masses associées à (2), le double de celles issues de (1) mais en (1) et (2), nous disposons déjà, à l'évaporateur, respectivement d'un taux d'ammoniac liquide de $66 \%$ et $33 \%$.

De même, la proportionnalité annoncée par la figure se vérifie bien sur le tableau I.

3.1.3 Frigories produites par $\mathrm{kg}$ de $\mathrm{CaCl}_{2}$. Le tableau II précise la quantité brute de kilofrigories disponible par $\mathrm{kg}$ de $\mathrm{CaCl}_{2}$ dans les mêmes conditions sous réserve d'une absorption complète en 18 heures pour revenir à l'état initial.

Tableau II.

$\begin{array}{cccrrrr}\theta & 90 & 100 & 110 & 120 & 130 & 140 \\ \mathrm{CaCl}_{2}, 8 \mathrm{NH}_{3} & 71 & -85 & -100 & -111 & -124 & -141 \\ \mathrm{CaCl}_{2}, 6 \mathrm{NH}_{3} & 32 & 54 & 63 & 72 & 79 & 89 \\ \mathrm{CaCl}_{2}, 4 \mathrm{NH}_{3} & 5,1 & 19 & 30 & 39 & 48 & 59\end{array}$

L'étude parallèle, menée sur la vaporisation nocturne avec reformation des ammoniacates, nous renseignera sur la valeur de cette limite.

L'intérêt de l'étude thermocinétique apparaît clairement par comparaison des résultats obtenus avec ceux que laissait prévoir la thermostatique sur le diagramme $(\log P, 1 / T)$.

En effet, 5 heures de chauffage de l'octoammoniacate ne permettent pas d'atteindre plus de $66 \%$ de la quantité d'ammoniac disponible.

3.1.4 Vitesse instantanée de libération de $\mathrm{NH}_{3}$ par $\mathrm{kg}$ de $\mathrm{CaCl}_{2}$. - Nous pouvons l'exprimer en taux \% d'ammoniac condensé pour les températures extrêmes soit 90 et $140^{\circ} \mathrm{C}$. Le tableau III indique pour les trois concentrations initiales des ammoniacates, les taux horaires en $\mathrm{NH}_{3}$ :

Tableau III.

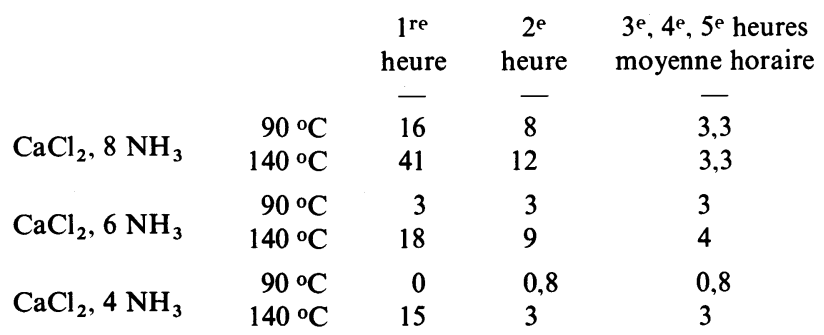

La richesse initiale du binaire est déterminée par une dissociation ou une régénération préalable destinée à lui donner la composition recherchée. L'opération est particulièrement simple : il suffit de lire à l'évaporateur le niveau libre étalonné d'ammoniac liquide dont la valeur renseigne sur le taux de l'ammoniacate contenu dans le générateur.

Observons qu'en général, durant la première heure, la vitesse de dissociation des ammoniacates est la plus forte. Ceci s'explique par le fait que la concentration initiale est maximale et par la nécessité de maintenir des conditions de départ parfaitement définies, c'est-à-dire de porter les ammoniacates à la température de dissociation en isolant le générateur et supprimant la condensation d'ammoniac durant le préchauffage.

Pendant les deux premières heures, la vitesse croît avec la température et la concentration initiales. Elle est sensiblement constante (3-4\%) durant les trois dernières heures de dissociation isotherme, ceci à toute température et pour une concentration quelconque. Une seule exception, l'ammoniacate pauvre dissocié isothermiquement à $90^{\circ} \mathrm{C}$, température à laquelle il n'évolue que faiblement durant tout le traitement : 0 à $0,8 \%$.

Ces observations montrent que les premières heures sont décisives puisque sur 5 heures de chauffe, durant les 2 premières heures soit $40 \%$ du temps, la condensation de $\mathrm{NH}_{3}$ correspond de 60 à $85 \%$ de la quantité condensable. En fin d'après-midi, une heure de chauffe en moins n'affecte le résultat que d'environ $3 \%$. La température du condensateur s'élevant au cours 
de la journée par suite de la condensation d'ammoniac explique cette évolution.

\subsection{COMPORTEMENT NOCTURNE. - Réfrigération} par évaporation d'ammoniac et régénération des ammoniacates. - Cette opération dure pratiquement 18 heures. Au laboratoire, nous avons pris 24 heures comme unité de durée afin de rendre les mesures cohérentes, mais l'examen du niveau de $\mathrm{NH}_{3}$ permet d'apprécier la quantité disparue en 18 heures, la température finale d'absorption étant voisine de $40^{\circ} \mathrm{C}$. En effet, si l'ammoniacate se refroidit rapidement de $140^{\circ} \mathrm{C}$ à $70^{\circ} \mathrm{C}$, la chaleur dégagée à la régénération maintient l'ammoniacate durant plus de 15 heures à une température comprise entre 70 et $40^{\circ} \mathrm{C}$. Il y a en réalité stockage thermique durant la nuit, phénomène utilisable si l'on recherche un chauffage nocturne, ce qui pourrait être le cas dans les régions désertiques.

Les nouvelles conditions initiales correspondent aux états du système à la fin des précédentes transformations. Le problème est donc de suivre le comportement de l'ensemble dans les 3 cas retenus précédemment. Les résultats sont consignés sur les figures 2 , 3 et 4 . Elles montrent comment, après 18 heures et 24 heures, l'ammoniac, libéré durant la journée, est réabsorbé la nuit en produisant du froid dans l'évaporateur. La figure 5 donne un exemple de variation en fonction du temps du volume d'ammoniac présent dans l'évaporateur.

Deux éléments sont à retenir :

- La quantité de $\mathrm{NH}_{3}$ évaporée.

- La capacité de réfrigération de cette évaporation associée au niveau de froid recherché. D'après la loi de Newton, les fuites thermiques sont proportionnelles à l'écart de température entre l'ambiance extérieure et celle de l'évaporateur. Ainsi la quantité des frigories disponibles varie en raison inverse de leur qualité. Aux environs de $-5^{\circ} \mathrm{C}$ à l'évaporateur le rendement en quantité est beaucoup plus faible qu'à $+5^{\circ} \mathrm{C}$ mais la qualité des frigories obtenues augmente.

Or, dans la réalité, le générateur est chauffé directement ou indirectement par le rayonnement solaire. Cet apport énergétique du rayonnement est une donnée physique sur laquelle on ne peut intervenir. Ceci entraîne 2 conséquences pratiques :

- la nécessaire réduction des fuites thermiques par une isolation soignée,

- la faible capacité de réfrigération lorsque le rayonnement incident sera réduit.

Le tableau IV et les figures 2,3 et 4 montrent qu'un apport énergétique diurne sensiblement constant libère d'autant plus d'ammoniac que la richesse initiale de l'ammoniacate est importante mais que l'absorption nocturne fixe des quantités de gaz du même ordre de grandeur avec - à une exception près à $90^{\circ} \mathrm{C}$ - un maximum caractérisant le couple initial de richesse moyenne. Par contre si l'on se fixe sur la qualité du froid obtenu, les paramètres cinétiques deviennent prépondérants et, comme on pouvait le prévoir, l'efficacité du couple initial pauvre est la plus forte. Cette dernière a été mesurée à partir du refroidissement de 121 d'eau contenus dans l'évaporateur. Nous constatons qu'en fin de nuit nous

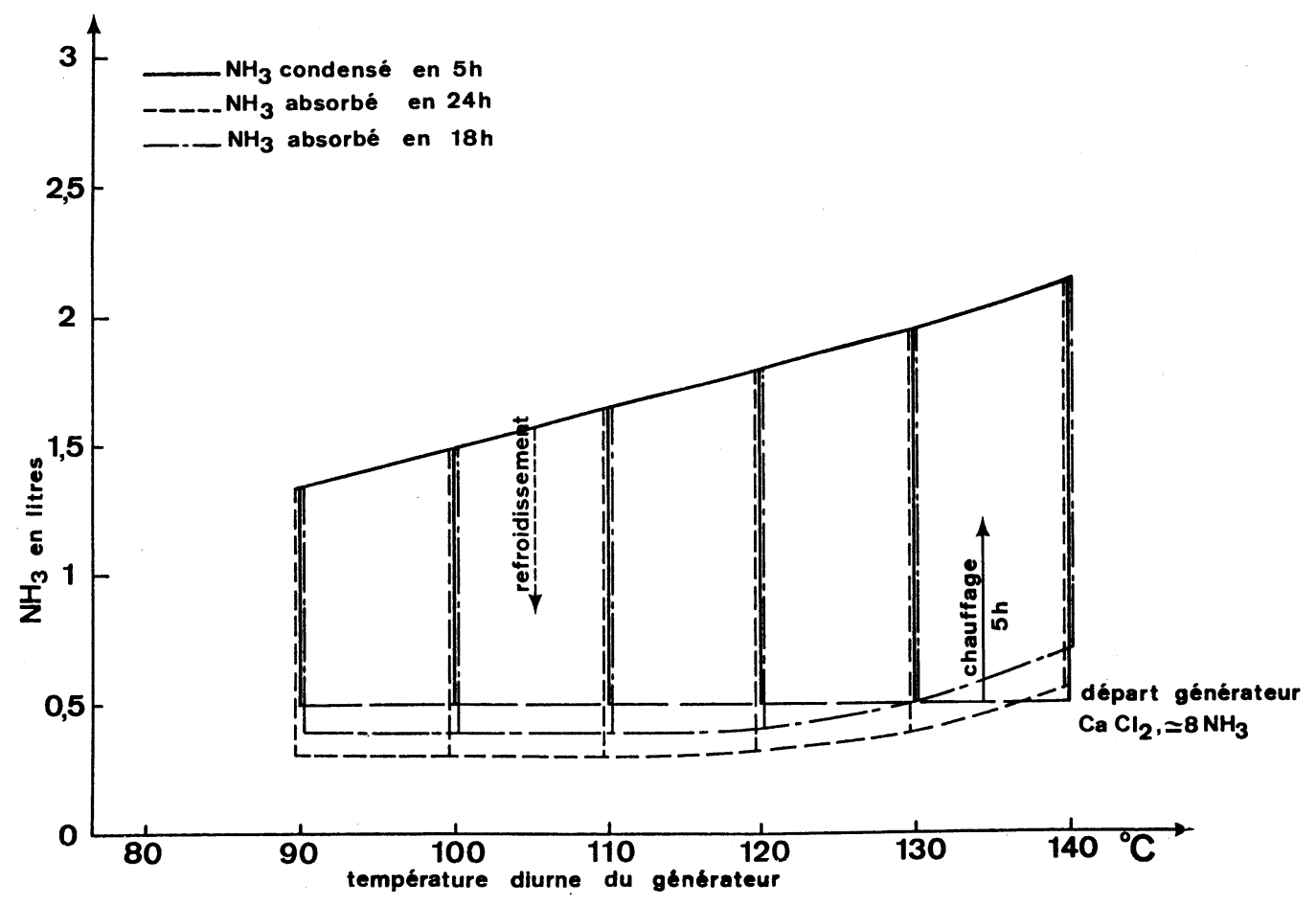

Fig. 2. - Régénération nocturne des ammoniacates après dissociation de l'octoammoniacate.

[Nocturnal regeneration of the ammoniacates after dissociation of the octoammoniacate.] 


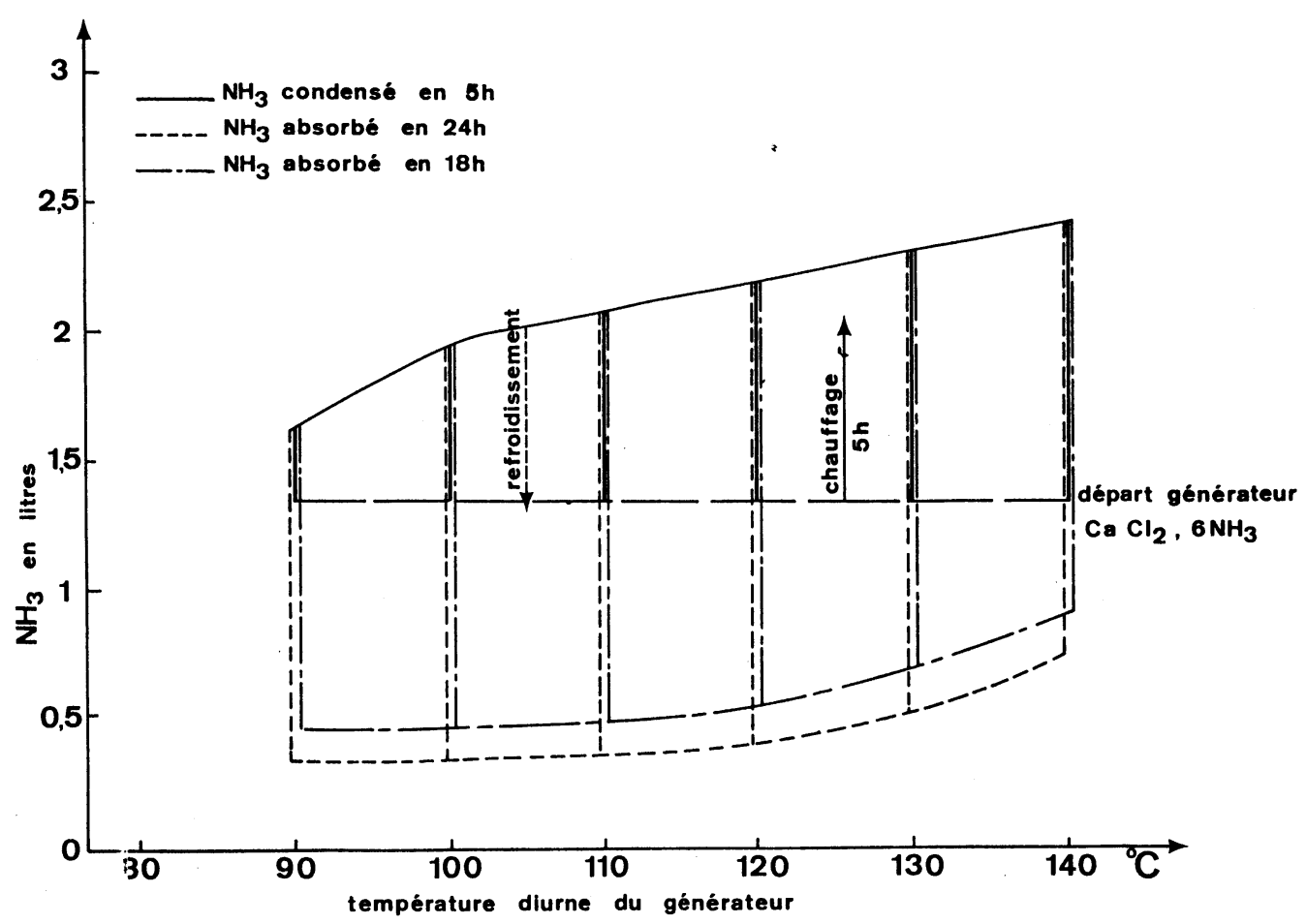

Fig. 3. - Régénération nocturne des ammoniacates après dissociation de l'hexammoniacate.

[Nocturnal regeneration of the ammoniacates after dissociation of the hexammoniacate.]

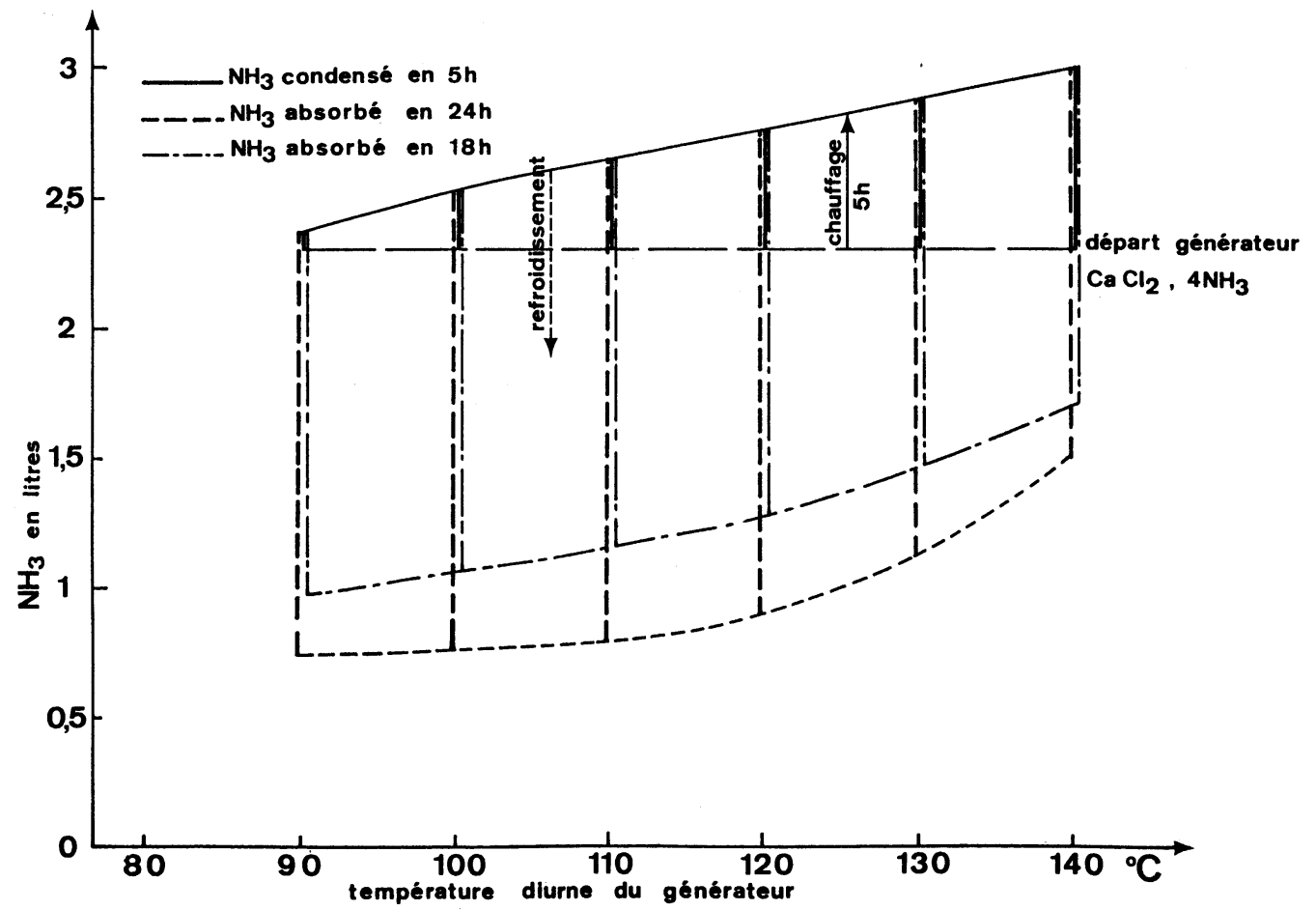

Fig. 4. - Régénération nocturne des ammoniacates après dissociation du tétrammoniacate.

[Nocturnal regeneration of the ammoniacates after dissociation of the tetrammoniacate.] 


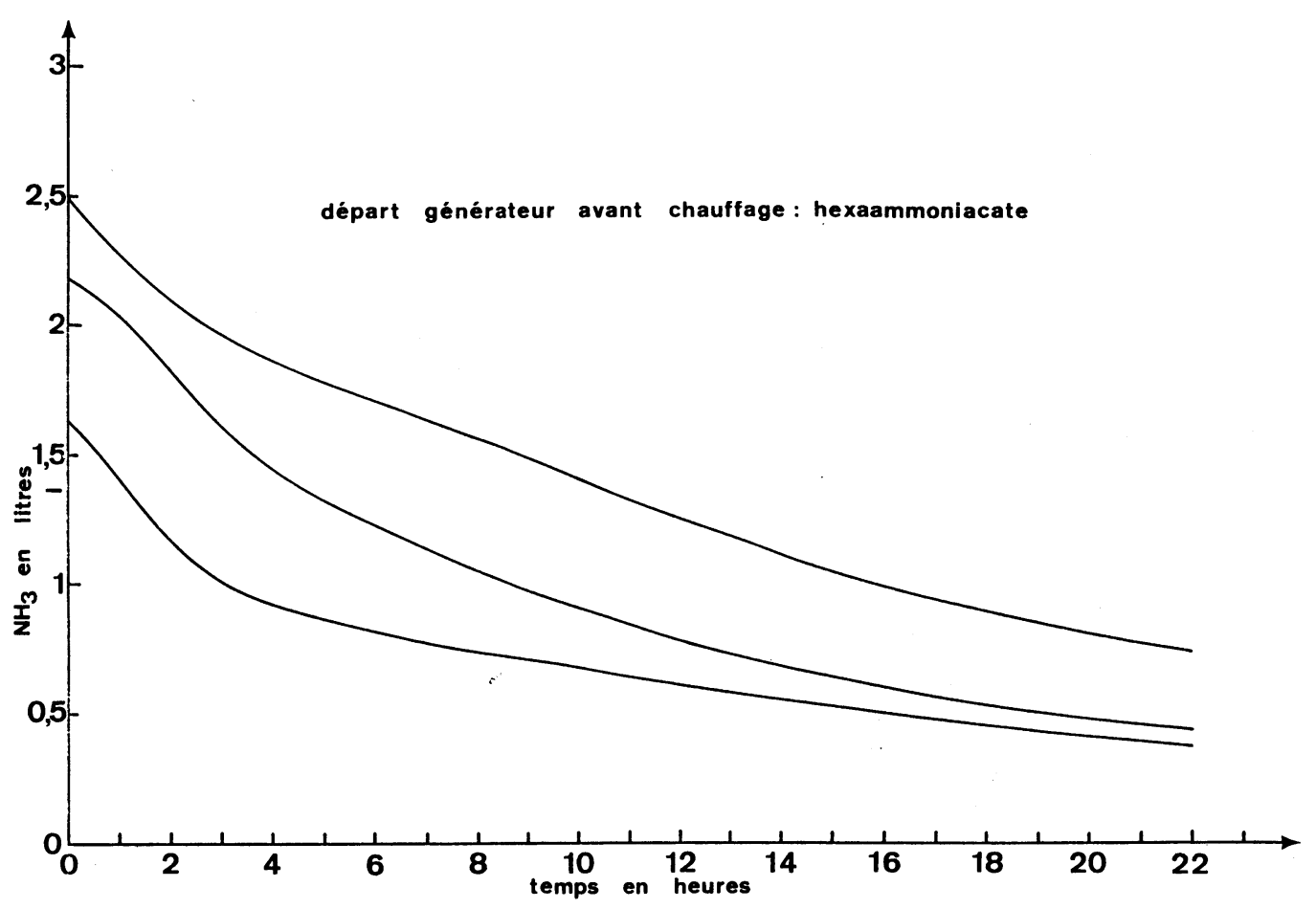

Fig. 5. - Variation du niveau libre de l'ammoniac dans l'évaporateur au cours de l'évaporation nocturne.

[Volume's variation of the ammonia in the evaporator for the nocturnal evaporation.]

retrouverons, pour la dissociation suivante, un nouvel état initial plus proche de $\mathrm{CaCl}_{2}, 8 \mathrm{NH}_{3}$ que des deux autres ammoniacates, ce qui signifie que le système se retrouvera rapidement et continûment dans le cas de la figure 2. La réabsorption nocturne est convenable.

Par exemple. - L'état final au lever du jour est voisin de $\mathrm{CaCl}_{2}, 8 \mathrm{NH}_{3}$, la vitesse de réabsorption devient très faible et compense à peine les inévitables fuites thermiques. La température de l'évaporateur ne baisse plus et même peut s'élever légèrement.

Les figures 7 et 8 montrent comment durant la nuit évoluent les températures de l'évaporateur et du générateur ainsi que le volume d'ammoniac liquide au cours de sa vaporisation.

Nous observons en particulier (tableau IV) qu'une même quantité d'ammoniac liquide peut conduire à des capacités de réfrigération fort différentes : elles sont d'autant plus élevées que la réabsorption est produite par un mélange solide éloigné de sa saturation en $\mathrm{NH}_{3}$.
3.3 CONSIDÉRATIONS CINÉTIQUES SUR LA RÉGÉNÉRATION DES ammONiaCATES. - Plank et Kuprianoff [12], examinant la régénération des ammoniacates à $T$ et $P$ constants, considèrent la réaction comme monomoléculaire et introduisent le temps $z_{0}$ de demi-réaction correspondant à la fixation de $2 \mathrm{NH}_{3}$, lorsque l'état initial est $\mathrm{CaCl}_{2}, 4 \mathrm{NH}_{3}$, et l'état final $\mathrm{CaCl}_{2}, 8 \mathrm{NH}_{3}$.

Ils notent alors que la fixation de $1 \mathrm{NH}_{3}$ supplémentaire exige un temps égal à $z_{0}$, celle de $\mathrm{NH}_{0} / 2$ suivant correspond encore à $z_{0}$, et ainsi de suite. Ils définissent alors le temps de demi-réaction réciproque $10^{3} / z_{0}$ minutes, grandeur qui permet de conduire pratiquement les calculs de quantité d'ammoniac effectivement fixée dans des conditions données.

En fait, il est aisé de généraliser ces observations en présentant le problème sous la forme suivante : si $G$ est la quantité d'ammoniac absorbé à l'instant $z$, $G_{\mathrm{s}}$, la quantité d'ammoniac absorbé après un temps infini, l'hypothèse la plus simple, liant $G$ à $z$, consiste à écrire que la variation relative de la différence

Tableau IV. - (1) Quantité $\mathrm{NH}_{3}$ absorbée en 18 heures, (2) frigories nettes obtenues à l'évaporateur.

$\theta$

\begin{tabular}{|c|c|c|c|c|c|c|c|c|c|c|c|c|}
\hline \multirow{3}{*}{$\begin{array}{c}\text { Couple initial } \\
-\end{array}$} & \multirow{2}{*}{\multicolumn{2}{|c|}{90}} & \multicolumn{2}{|c|}{100} & \multicolumn{2}{|c|}{110} & \multicolumn{2}{|c|}{120} & \multicolumn{2}{|c|}{130} & \multicolumn{2}{|c|}{140} \\
\hline & & & & & & & & & & & & \\
\hline & (1) & (2) & (1) & (2) & (1) & (2) & (1) & (2) & (1) & (2) & (1) & (2) \\
\hline $\mathrm{CaCl}_{2}, 8 \mathrm{NH}_{3}$ & 0,96 & 40 & 0,97 & 31,2 & 1,3 & 43,6 & 1,56 & 44,8 & 1,49 & 50,4 & 1,42 & 49.2 \\
\hline $\mathrm{CaCl}_{2}, 6 \mathrm{NH}_{3}$ & 1,15 & 41,2 & 1,5 & 46 & 1.58 & 48 & 1,61 & 59,2 & 1,58 & 62,4 & 1,50 & 70 \\
\hline $\mathrm{CaCl}_{2}, 4 \mathrm{NH}_{3}$ & 1,41 & 62 & 1,45 & 57,2 & 1,38 & 67,2 & 1,48 & 67,2 & 1,42 & 67,2 & 1,33 & 66,8 \\
\hline
\end{tabular}


$G_{\mathrm{s}}-G$ durant le temps $\mathrm{d} z$ est proportionnelle à $\mathrm{d} z$ :

$$
\frac{\mathrm{d}\left(G-G_{\mathrm{s}}\right)}{G-G_{\mathrm{s}}}=-k \mathrm{~d} z
$$

loi aisément vérifiable sous la forme :

$$
\log \left(G_{\mathrm{s}}-G\right)=\log G_{\mathrm{s}}-k z .
$$

Or si l'on observe les conditions de fonctionnement du réfrigérateur expérimental durant les 18 heures de régénération des ammoniacates on note que la température du générateur chute très rapidement de $140^{\circ} \mathrm{C}$ à $50^{\circ} \mathrm{C}$ puis évolue lentement vers $40^{\circ} \mathrm{C}$ en fin d'absorption car la chaleur de formation des ammoniacates maintient une relative constance de la température au générateur. D'autre part, le diagramme $(1 / T, \log P)$ de $\mathrm{CaCl}_{2}, \mathrm{NH}_{3}$ [4] montre qu'à $50^{\circ} \mathrm{C}$, la pression d'équilibre de $\mathrm{NH}_{3}$ sur le couple (riche) à $49,9 \%$ est de 3 bars, valeur nettement inférieure à $P_{\mathrm{m}} \mathrm{NH}_{3}$ à $0^{\circ} \mathrm{C}$. Le générateur étant constamment en communication avec l'évaporateur durant l'absorption et ce dernier contenant $\mathrm{NH}_{3}$ liquide en équilibre avec sa vapeur, nous pouvons affirmer que durant la phase active de réfrigération, c'est l'évaporateur et non le générateur qui impose la pression de travail à l'ensemble.

Finalement, pour des raisons pratiques évidentes, nous cherchons à maintenir aussi constante que possible la température de l'évaporateur donc la pression au générateur durant la régénération. Ainsi la loi précédente doit, en première approximation, s'appliquer. C'est ce que nous vérifions à partir de la figure 5 consignant les niveaux libres de $\mathrm{NH}_{3}$ en fonction du temps, sur la figure 6 où l'on porte en fonction du temps le logarithme de la différence des volumes de $\mathrm{NH}_{3}$ liquide absorbés en temps infini et à l'instant $z$.

4. Etude énergétique. - 4.1 GÉNÉRALITÉs. - Le rythme, 6 heures de chauffe ou condensation, 18 heures de refroidissement ou évaporation est impératif, soit un cycle par 24 heures. Ceci entraine plusieurs conséquences essentielles, caractéristiques de la réfrigération solaire :

$1^{\circ}$ La nécessité d'employer une masse d'ammoniac liquide aussi importante que possible, donc une quantité correspondante d'absorbeur, ce qui entraînera un encombrement important des ensembles.

$2^{\circ} \mathrm{La}$ quasi-impossibilité de maintenir constante la température de l'évaporateur au cours du chauffage durant la journée, mais l'obligation d'employer un dispositif approprié réduisant l'amplitude des variations de température à des limites compatibles avec la conservation des denrées périssables placées dans l'armoire.

4.2 MÉTHODE. - Quelles que soient les dispositions retenues, l'intérêt du réfrigérateur se mesure à la quantité de frigories réellement disponibles dans l'évaporateur. Dans les conditions indiquées, nous disposons de $M=4 \mathrm{~kg}$ d'eau à l'intérieur du volume à refroidir et enregistrons leur température $\theta$. Si $\left(\theta_{1}-\theta_{2}\right)$ est la différence observée entre 15 heures et 9 heures le lendemain, nous en tirons la capacité réelle de refroidissement $F$ de notre dispositif :

$$
F=M\left(\theta_{1}-\theta_{2}\right)
$$

$F$ pourra certainement être amélioré car l'évaporateur a été d'abord conçu en fonction d'un critère de sécu-

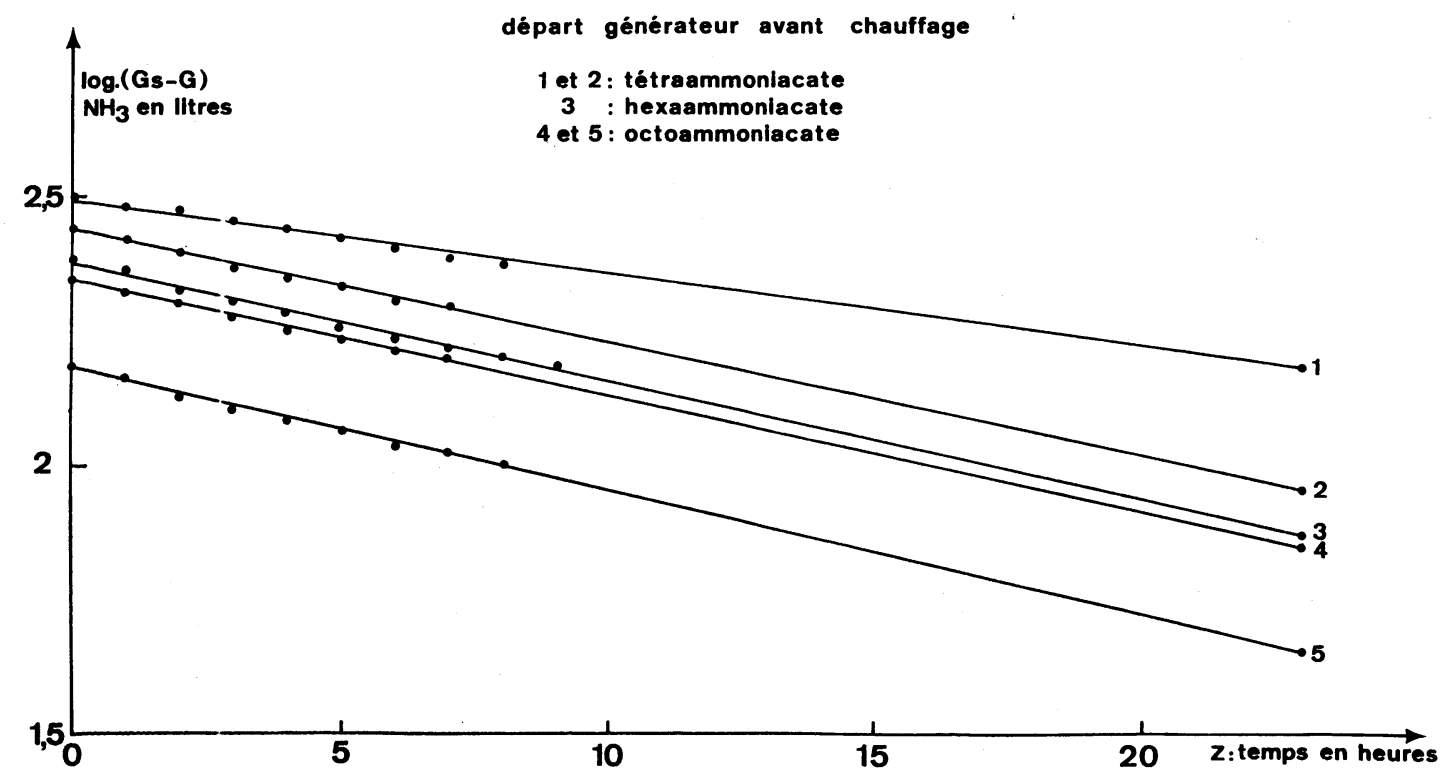

Fig. 6. - Cinétique de régénération des ammoniacates.

[Kinetic of regeneration of the ammoniacates.] 


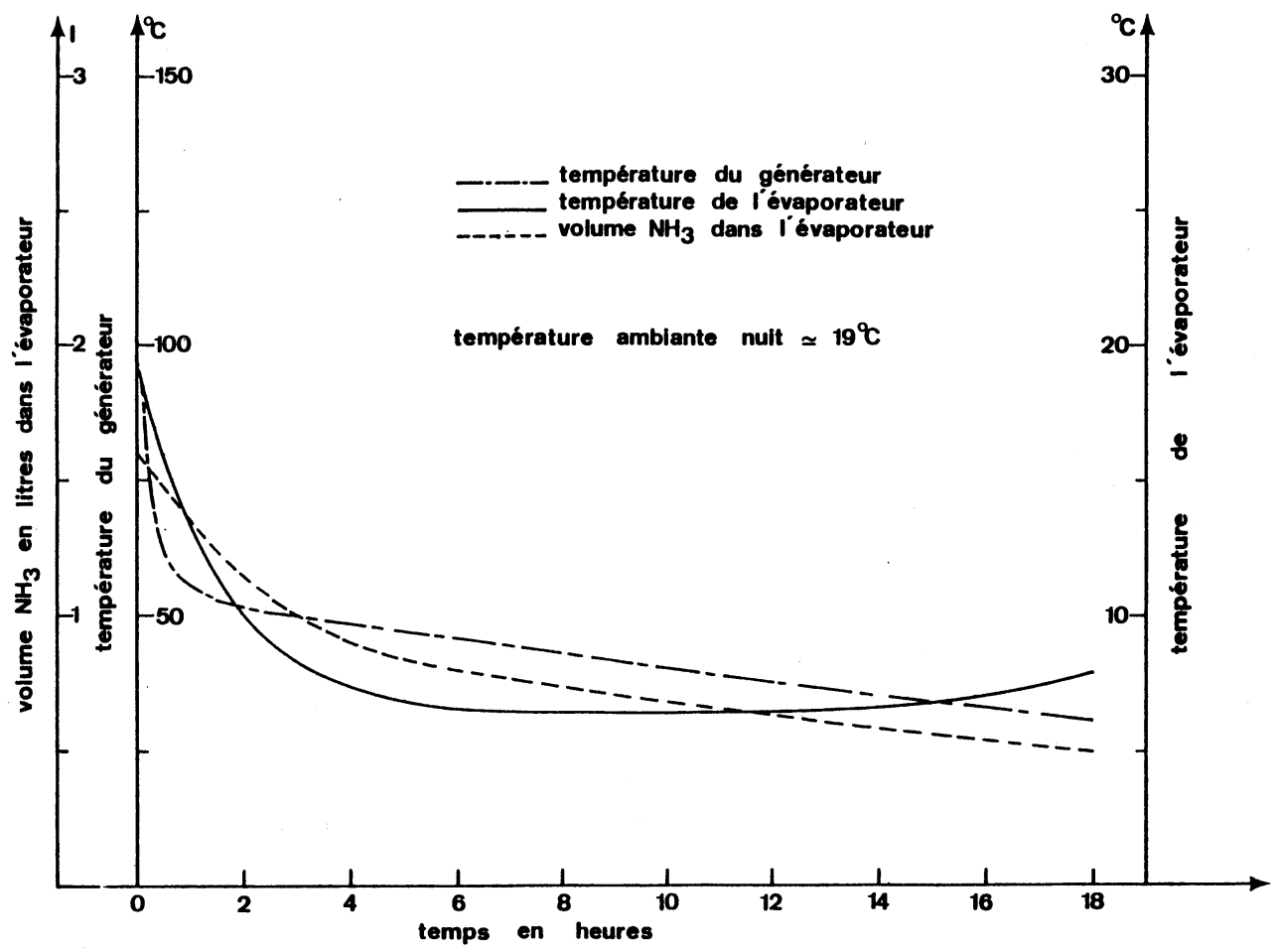

Fig. 7. - Comportement du réfrigérateur durant la phase de refroidissement. a) Couple pauvre en ammoniac.

[Deportment of the refrigerator for the phasis of the cooling. a) Couple poor in ammonia.]

rité pour le personnel : c'est un cylindre d'acier testé à 30 bars de contenance $5 \mathrm{l}$, isolé dans une enceinte d'environ 201 où les échanges thermiques se font par convection, conduction et rayonnement. Nous réduirons les masses et les épaisseurs à une valeur acceptable après conclusion des essais.

Or, si $V$ est le volume en litres de $\mathrm{NH}_{3}$ évaporé, nous pouvons calculer le rendement brut $\rho_{\mathrm{B}}$ de l'évaporateur :

$$
\rho_{\mathrm{B}}=\frac{F}{V .180}=\frac{M\left(\theta_{1}-\theta_{2}\right)}{V .180}
$$

puisqu'un litre de $\mathrm{NH}_{3}$ évaporé dans les conditions de l'expérience correspond sensiblement à $180 \mathrm{kcal}$.

Le rendement énergétique total :

$$
\rho_{\mathrm{t}}=\frac{F}{W_{\text {elect }}}=\frac{M\left(\theta_{1}-\theta_{2}\right) 4185}{V_{\text {eff }} \cdot I_{\text {eff }} \cdot t_{\mathrm{s}}} .
$$

Le tableau IV résume les expériences faites avec leurs performances.

4.3 OBSERVATIONS. - Dans les conditions étudiées, le rendement total $\rho_{\mathrm{t}}$ varie de 2 à $5 \%$. Notons que le rendement solaire réel sera encore inférieur car l'intensité rayonnante type par ciel clair n'est que rarement atteinte, ce qui signifie que la quantité d’ammoniac condensé au cours de la journée sera inférieure.

Le rendement brut de réfrigération se situe aux environs de $20 \% .4$ sur 5 des frigories produites sont absorbées pour le refroidissement des appareils et par les fuites thermiques.

Les vitesses de réaction conditionnent absolument les rendements énergétiques. Nous limitant aux résultats que laissaient prévoir les réactions à l'équilibre, nous trouvions que $1 \mathrm{~kg} \mathrm{de} \mathrm{CaCl}_{2}$ permettait de travailler avec $0,92 \mathrm{~kg}$ d'ammoniac liquide, soit 1,51 mesuré dans les conditions habituelles. Or, le rythme imposé par la succession quotidienne des cycles limite à 6 heures la durée totale du chauffage à partir de l'ambiante, ce qui à $140{ }^{\circ} \mathrm{C}$ libère environ $50 \%$ de la quantité théorique quel que soit le type de générateur car nous pouvons monter rapidement à cette température et nous assurons, par un thermomètre placé au cœur de la masse chauffée, que tout le matériau est alors en équilibre thermique. De même en 18 heures la réabsorption ne correspond pas à plus de $60 \%$ de la quantité que pourrait fixer $\mathrm{CaCl}_{2}$ en temps suffisant.

Ainsi s'expliquent les écarts importants - près de $70 \%$ - observés entre l'expérience conduite à température et en temps réels et les prévisions que laissaient espérer les propriétés chimiques du sel. Naturellement, ces remarques sont valables pour tous les couples frigorigènes. Elles incitent à une certaine prudence dans l'estimation des possibilités de l'utilisation de l'énergie solaire et légitiment la pratique d'expériences préliminaires réalistes avant le passage à l'exploitation industrielle ou domestique. 


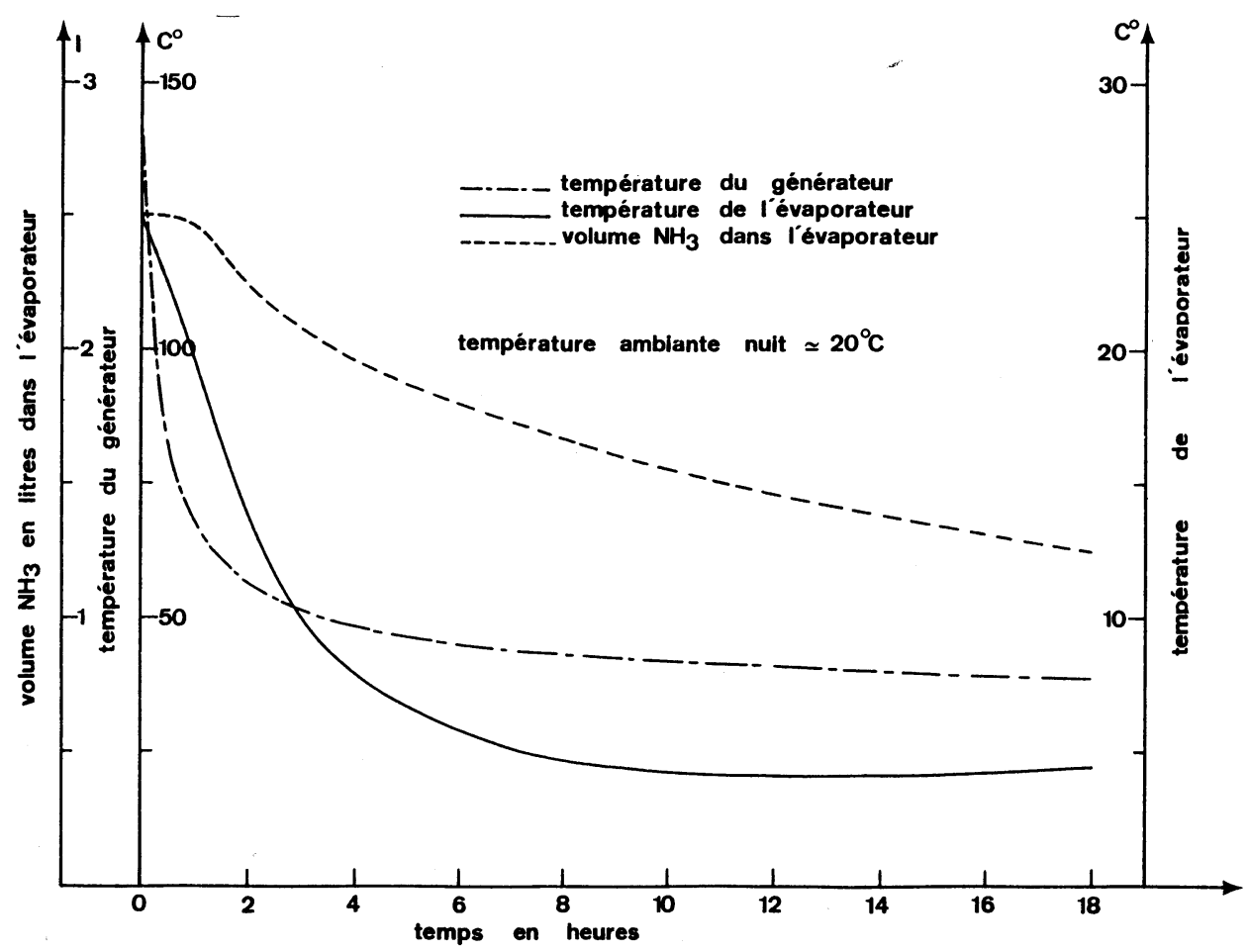

Fig. 8. - Comportement du réfrigérateur durant la phase de refroidissement. $b$ ) Couple moyen.

[Deportment of the refrigerator for the phasis of the cooling. $b$ ) Couple mean.]

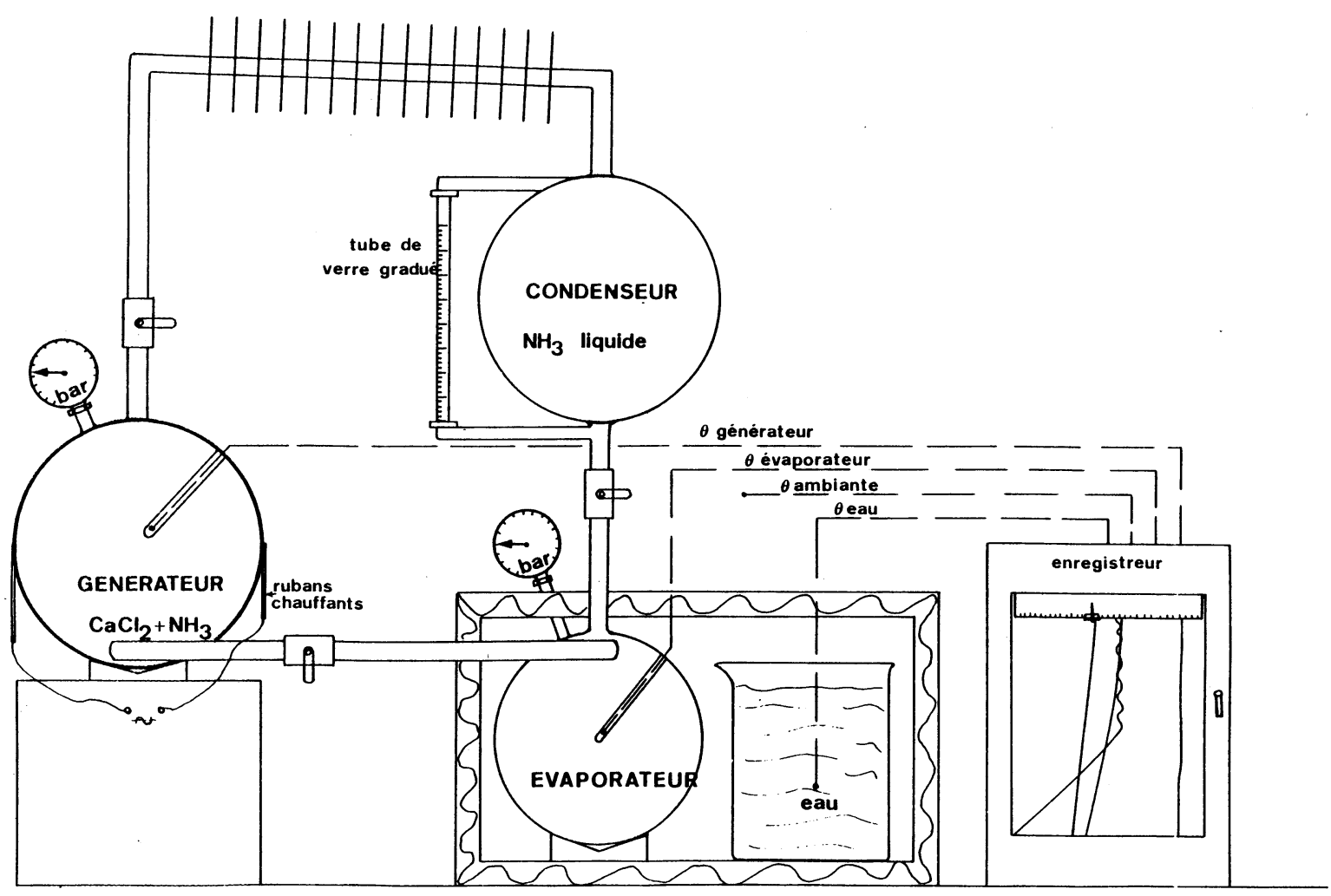

Fig. 9. - Montage utilisé au laboratoire.

[Apparatus used in our laboratory.] 
5. Conclusions. - Retenons d'abord le trait commun au chauffage et à la réfrigération solaires : pour obtenir des services analogues, les radiateurs comme les évaporateurs doivent être surdimensionnés si on les compare aux appareils classiques; on ne peut, en effet, que recueillir l'énergie incidente sans pouvoir la modifier au gré des besoins.

Les masses de liquide frigorigène et d'absorbant seront donc importantes si l'on veut obtenir une quantité de frigories suffisante pour les rendre pratiquement utilisables. Si l'on se réfère aux normes communément admises [13] une armoire domestique exige en moyenne 1200 frigories nettes par 24 heures, soit à 100 frigories $/ \mathrm{kg}$ de $\mathrm{NH}_{3} 12 \mathrm{~kg}$ de fluide donc, environ 201 qui représentent le volume minimum du stockage au condenseur.

D'un autre point de vue, 1200 frigories nettes représentent au mieux $5 \%$ de l'énergie journalière incidente, soit $2400 \mathrm{kcal}$, c'est-à-dire $28 \mathrm{kWh}$ ce qui exige une surface captante de $5 \mathrm{~m}^{2}$ si l'énergie journalière reçue par mètre carré est $6 \mathrm{kWh}$. L'encombrement des ensembles s'en déduit immédiatement.

La réduction des variations diurnes de température à l'évaporateur est indispensable à la conservation des denrées périssables.

L'intérêt de la simulation, que nous avons conduite, nous apparaît maintenant clairement : si la thermostatique confirmait les prévisions brutes de la stæchiométrie $\left(6 \mathrm{NH}_{3}\right.$ libérés par mole de $\left.\mathrm{CaCl}_{2}\right)$, soit 1,51 d'ammoniac liquide par $\mathrm{kg}$ de $\mathrm{CaCl}_{2}$, la limite supérieure des quantités réellement disponibles de liquide frigorigène n'excédera pas 0,51 par $\mathrm{kg}$ de sel, soit $30 \%$ de la quantité totale.

Les masses d'absorbant et de frigorigène nécessairement présentes et nécessairement inutilisables par suite de la rigueur du rythme imposé par le soleil et les exigences de la cinétique physico-chimique, seront donc très importantes.

\section{Bibliographie}

[1] Flechon, J., Martin, G., Toure, I., C.R. Hebd. Séan. Acad. Sci. 280B (1975) 211.

[2] Flechon, J., C.R. Hebd. Séan. Acad. Sci. 283B (1976) 127.

[3] Flechon, J., Revue Phys. Appl. 11 (1976) 743-50.

[4] Flechon, J. et Machizaud, F., Revue Phys. Appl. 14 (1979) 97-105.

[5] Plank, R. and Kuprianoff, J., die Kleinkältemaschine (Spring Verlag, Berlin) 1960 , p. 351.

[6] Linge, K., Über periodische Absorptionkältemaschinen, D. Sc. Thesis Karlsruhe, May 1929.

[7] AltenKIRSCH, E., Absorptionskältemaschinen (Verlag Technik, Berlin) 1954.

[8] MenL, W., Die thermischen Eigenschaften Zeitschrift für die gesamte Kälteindustrie (Heft 11) 1934, Vol. 41, p. 186.
[9] Bonauguri, E., Preliminary notes on a solar Absorption Unit, Proceedings of the 12th International Congress of refrigeration, I.F.F., Paris (1969) 1411.

[10] Muradov, D. and Shadiev, O., "Intermittent solar refrigerator with solid absorbent », Izd, Tasch, G.V. i B.G.P.I., Tashkent (1969).

[11] Shadiev, O. and Umarov, G. Y., "On the temperature Distribution in a solar Refrigerator Generator and the thermal conductivity of the absorbent ", Geliotekhnika 8 (1974) 34.

[12] Plank et Kuprianoff, ouvrage cité, p. 342.

[13] Degorn, Réfrigération électrique, Automatique (Girardot), épuisé. 